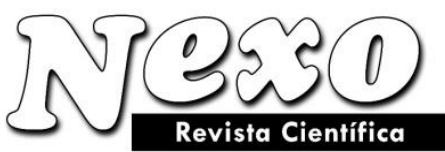

Vol. 34, No. 02, pp. 534-546/Mes 2021

\title{
Potencial del laboratorio de biotecnología del PIESA-UNI para desarrollar bioprocesos ambientales, agrícolas e industriales.
}

\section{Biotechnological potential from PIESA-UNI laboratory to development environmental, agricultural and industrial bioprocesses.}

\author{
Leandro Alberto Páramo Aguilera*, Heysell Dodanig Delgado Silva, Cesia K. Ríos Guevara \\ Universidad Nacional de Ingeniería. Programa de Investigación Estudios Nacionales y Servicios del \\ Ambiente. Managua, Nicaragua. \\ *Iparamo2014@gmail.com ; dodanig94@yahoo.es
}

(recibido/received: 01-septiembre-2020; aceptado/accepted: 15-diciembre-2020)

\section{RESUMEN}

Los microorganismos se encuentran presentes en todos los ambientes y son capaces de sintetizar productos útiles para el hombre, representan unos pocos centenares de especies de entre las más de cien mil descritas en la naturaleza. La biotecnología tiene un carácter interdisciplinario y por medio de sus herramientas, permite la obtención de productos a partir de materias primas, incidiendo sus aplicaciones sobre alimentos, industria agroalimentaria, medicina, producción industrial, medio ambiente y producción de energía, siendo el uso de microorganismos una constante en la mayoría de las áreas. La importancia de la biotecnología blanca radica en el diseño de productos y la aplicación de procesos biotecnológicos como alternativa a procesos químicos convencionales, que conlleven ventajas económicas y medioambientales y reduzcan o eliminen el uso y generación de sustancias peligrosas. El presente trabajo, resume la investigación de varios años desarrollada en el PIENSA-UNI, con el propósito de colectar microorganismos provenientes de diferentes fuentes, aislarlos e identificarlos por vía molecular, para finalmente desarrollar bioprocesos que contribuyan a la solución de problemas ambientales (biorremediación de metales pesados, de suelos, de pesticidas, de petróleo, biolixiviacion, manejo de residuales industriales), desarrollo de aplicaciones agrícolas (estimuladores del crecimiento, biofertilizantes, bioinsecticidas) e industriales como producción de diferentes enzimas.

Palabras claves: Biofertilizantes, biodegradación, biorremediación, Producción de enzimas, Desarrollo de Bioprocesos.

\begin{abstract}
Micro-organisms are present in all environments and are capable of synthesizing products useful to man, they represent a few hundred species among the more than one hundred thousand described in nature. Biotechnology has an interdisciplinary character and through its tools, allows the production of products from raw materials, impacting their applications on food, agri-food industry, medicine, industrial production, environment and energy production, the use of micro-organisms being a constant in most areas. The importance of white biotechnology lies in the design of products and the application of biotechnological processes as an alternative to conventional chemical processes, which bring economic and environmental
\end{abstract}


benefits and reduce or eliminate the use and generation of hazardous substances. The present work summarizes the research of several years developed in the PIENSA-UNI, with the purpose of collecting microorganisms from different sources, isolating them and identifying them by molecular way and finally to develop bioprocesses that contribute to the solution of environmental problems (bioremediation of heavy metals, soils, pesticides, petroleum, biolixiviation, industrial waste management), development of agricultural applications (growth stimulators, biofertilizers, bioinsecticides) and industrial as production of different enzymes.

Keywords: Biofertilizer, biodegradation, bioremediation, enzyme production, Development of Bioprocesses.

\section{INTRODUCCIÓN}

La biotecnología ambiental está íntimamente relacionada con la bioprospección microbiana ya que esta se basa en el estudio y gestión de las comunidades de microorganismos para obtener productos de calidad y proporcionar servicios a la comunidad y al ambiente. Por otra parte, la biotecnología agrícola tiene por objeto mejorar la calidad, el rendimiento y reducir al mínimo el uso de prácticas agrícolas tradicionales que consumen energía y contaminan el ambiente. La biotecnología industrial, también conocida como biotecnología blanca, se relaciona con la utilización de sistemas biológicos para la fabricación, transformación o degradación de moléculas, gracias a procesos enzimáticos y fermentativos. La biotecnología ha encontrado su aplicación en sectores tan diversos como la agricultura, medicina, medio ambiente e industria (Huete y Orozco, 2001). Su desarrollo en Nicaragua es muy incipiente y existe una baja capacidad en cuanto a infraestructura y equipos, tanto en el ámbito investigativo como productivo. Sin embargo, hay que destacar que, en los últimos años, esta área tecnológica se ha visto fortalecida en cuanto a la formación de recursos humanos (Loásiga \& Cisne, 2001). La Universidad Nacional de Ingeniería mediante el programa de investigación y estudios nacionales sobre el ambiente (PIENSA), ha realizado desde sus orígenes diversos trabajos de investigación (Blanco y Lanza., 2001; Toruño y Escobar., 2002) los cuales, a pesar de no contar con un enfoque biotecnológico, han sido capaces de brindar una solución biológica a ciertos problemas relacionados con el ambiente.

La biotecnología verde es aquella aplicada a la agricultura, ejemplo de ello es el diseño de plantas transgénicas capaces de crecer en condiciones ambientales desfavorables, plantas resistentes a plagas, a enfermedades, cultivos mejorados y el desarrollo de usos no alimentarios de los cultivos, como la producción de biocombustibles, en todos estos procesos participan también los microrganismos. Aceves y Castañeda, (2012), efectuaron una revisión bibliográfica para la producción biotecnológica de lipasas microbianas como una alternativa sostenible para la utilización de residuos agroindustriales. Pedraza, et al., (2010), concentran su investigación en temas relacionados con aspectos inherentes a las interacciones biológicas que se dan en el suelo y su efecto sobre el crecimiento de las plantas y la conservación del ambiente, además de la importancia de los microorganismos y su utilización biotecnológica.

Piñero, (2013) afirma que, según investigaciones realizadas en diferentes países Latinoamericanos, la Bioprospección microbiana ha resultado beneficiosa en la búsqueda de solución a problemas medio ambientales, así como una fuente de ingresos económicos (biorrecuperación de suelos, biosaneamiento de efluentes, enzimas de restricción y microorganismos usados como fijadores de nitrógeno). Cabe destacar que esta línea de investigación se encuentra dentro del marco de la biotecnología ambiental la cual no se ha explotado en todo su esplendor quedando como la última opción para la comunidad científica. Ante la situación planteada, en este proyecto se determinó como objetivo principal el aislamiento y caracterización morfológica y molecular de microorganismos provenientes de ríos, suelos y bosques de Nicaragua, además de empresas artesanales de procesamiento de queso y oro, para encontrar posteriormente aplicaciones que los mismos puedan tener en la solución de las distintas problemáticas medioambientales, agrícolas e 
industriales que afectan al país. Además, esta investigación pretende incentivar o promover mediante la investigación de la biotecnología, la Bioprospección en Nicaragua con el desarrollo de bioprocesos que coadyuven a la solución de los problemas más acuciantes que enfrentamos los nicaragüenses.

\section{METODOLOGÍA}

La biotecnología microbiana es el ámbito de la microbiología orientado a la producción de elementos de interés industrial mediante procesos en los cuales intervenga, en algún paso, un microorganismo. La mega tendencia que persiste desde la primera década del siglo XXI es la explosión de biotecnología y nanotecnología, optimización en el uso de agua y producción sustentable de alimentos. "La biotecnología es en estos momentos la manifestación más avanzada del conocimiento científico y tecnológico". Es una ciencia moderna que está revolucionando tanto a la industria como a la agricultura en determinadas zonas. Una de las principales características de esta ciencia es la forma en que está vinculada con el bienestar del hombre, con el medio ambiente y su sostenibilidad (González, 2012).

Como resultado de los trabajos de Bioprospección y de aplicación de lo bioprospectado e identificado a la fecha, desarrollados en los laboratorios del PIENSA, de la Universidad Nacional de Ingeniería de Nicaragua, actualmente se cuenta con alrededor del 400 aislados provenientes de diferentes lugares que han sido muestreados y que antes fueron indicados. De lo muestreado e identificado se tienen un poco más de 100 microorganismos con más del $60 \%$ identificados hasta el nivel de especie, por lo que aún resta por identificar más de 200 microorganismos de los cuales se espera poder determinar una gran cantidad de nuevos consorcios y microorganismos de interés para el desarrollo de múltiples bioprocesos, además de los mostrados aquí. Este trabajo muestra el enorme potencial con el que se cuenta como producto del desarrollo de proyectos de Bioprospección para el desarrollo de diversos bioprocesos, para la biorremediacion de suelos contaminados con petróleo, aguas contaminadas con diferentes metales pesados, biorremediacion en suelos contaminados con plaguicidas, bioinsecticidas, biofertilizantes y muchos otros procesos más. Obviamente que ahora contamos con el recurso biológico plenamente identificado, pero se requiere de recursos económicos para el desarrollo de estos bioproductos.

\section{Puntos de muestreo y lugar de realización.}

Para esta investigación se trabajó con muestras de suelo y agua provenientes de diferentes sitios contaminados, donde se pudre materia orgánica y que hasta la fecha existe poca incidencia humana. La recolección de muestras se realizó en los siguientes puntos: Reserva natural Chocoyero en Ticuantepe, Rio Chiquito en León, Minería y quesera artesanal en la Libertad, Chontales (Figura 1). La caracterización microbiológica de los microorganismos se trabajó en el laboratorio de biotecnología y microbiología del PIENSA-UNI. La extracción de ADN se realizó en el laboratorio de Biologia molecular de la Universidad Centroamericana (UCA) y la secuenciación se realizó en los laboratorios Macrogen de Corea del Sur (Delgado-Silva y Fonseca Cruz, 2019; Ríos-Guevara, 2019; Guillén-Rodríguez y Martínez-Laguna, 2019). Todos los trabajos antes citados, recogen en detalle el desarrollo de las diferentes técnicas utilizadas, desde el aislamiento microbiano, hasta la identificación microbiana haciendo uso de las herramientas moleculares. Para una síntesis de las técnicas utilizadas consúltese la referencia Páramo-Aguilera, et al., 2018. 


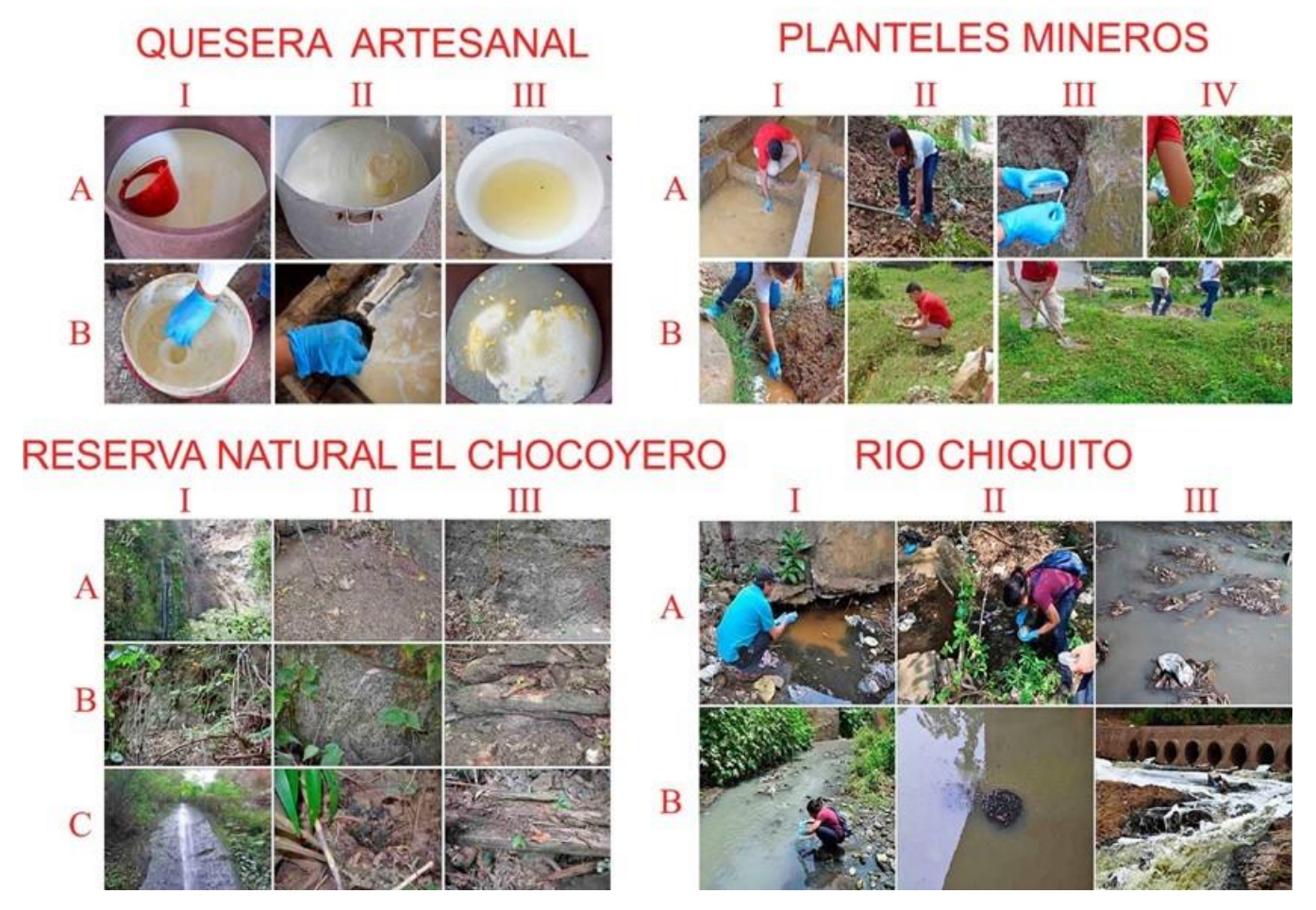

Figura 1. Se pueden apreciar los diferentes sitios muestreados y los puntos de los cuales fueron tomadas las muestras. Quesera artesanal (AI. Leche fresca, AII. Leche descremada, AIII. Lactosuero salado, BI. Lactosuero simple fermentado, BII. Sedimentos de drenaje, BIII. Aguas residuales), Planteles mineros (AI. Lodo en dique de cola, AII. Aguas residuales, AIII. Cola o lama fresca, AIV. Cola o lama vieja, BI. Aguas residuales estancadas, BII. Sedimentos de drenaje, BIII y BIV. Suelo de colas), ambas ubicadas en el municipio de La Libertad, Chontales. Reserva Natural El Chocoyero / El brujo ubicada en el Municipio de Ticuantepe, Departamento de Managua. (AI. Cascada El Chocoyero, AII. Suelo degradado, AIII. Roca recubierta de biopelícula blanca, BI. Roca recubierta de lama, BII. Roca recubierta de una flora blanca y verdosa, BIII. Tronco de un árbol degradado, CI. Cascada El Brujo, CII. Suelo degradado, CIII. Tronco de árbol degradado). Rio Chiquito de la ciudad de León (AI. Puente el Bypass, AII. Puente la vía 23 de Julio, AIII. Puente Martínez El Calvario, BI. Puente Tenería Los Leones, BII. Puente Camino al Fortín Tenerías, BIII. Puente pilas sépticas).

\section{Método}

Para el desarrollo de este trabajo se revisaron los trabajos monográficos desarrollados por los estudiantes graduandos de la carrera de ingeniería Química (Delgado-Silva y Fonseca-Cruz, 2019; Ríos-Guevara, 2019; Guillén-Rodríguez y Martínez-Laguna, 2019) que trataron en profundidad el tema de la Bioprospección microbiana con vistas a buscar aplicaciones diversas en la agricultura, la industria y el ambiente. Al mismo tiempo se revisaron trabajos desarrollados por O’Connor-Mendoza, 2019; Flores-Hernández y RoqueCastellano, 2017 y Méndez-Úbeda, 2018 y que trataron sobre el desarrollo de aplicaciones particulares de los microorganismos bioprospectados por ellos a partir de muestras de bioinsumos comerciales. Estos últimos autores en unión con Guillén-Rodríguez y Martínez-Laguna, 2019; propusieron y llevaron hasta el nivel de aplicación a invernadero bioprocesos para desarrollar la producción de un consorcio microbiano con fines de ser utilizado como biofertilizador y un bioinsecticida a base de Bacillus subtilis.

Todos los trabajos antes mencionados, han tenido en común, la aplicación de diversas metodologías entre las que podemos resumir: Toma de muestras (en bioinsumos artesanales, en una quesera artesanal y en una minera artesanal del municipio de la Libertad en Chontales, en la Reserva Biológica El Chocoyero/El Brujo en Ticuantepe y en el Rio Chiquito de la ciudad de León), aislamiento e identificación morfológica de las 
cepas obtenidas, identificación molecular de hongos y bacterias seleccionadas, la búsqueda en bases de datos internacionales de las diversas aplicaciones reportadas para el desarrollo de bioprocesos con los microorganismos identificados, pruebas de antagonismo microbiano, desarrollo de la cinética microbiana y producción a nivel de shacker en el laboratorio, aplicación a nivel de invernadero a diversos cultivos como la papa, el rábano, la chiltoma y el pepino, sobre los cuales se midieron y evaluaron distintas variables de interés agronómico y que permitieron evaluar la eficacia de los productos biotecnológicos desarrollados. El detalle de las metodologías a las que hacemos mención, pueden ser consultadas por medio de los trabajos antes citados.

\section{RESULTADOS Y DISCUSIÓN}

La biotecnología tiene un carácter interdisciplinario y por medio de la aplicación de métodos permite la obtención de productos a partir de materias primas, incidiendo sus aplicaciones sobre alimentos, industria agroalimentaria, medicina, producción industrial, medio ambiente y producción de energía, siendo el uso de microorganismos una constante en la mayoría de las áreas (González, 2011). Actualmente se han realizado diversas investigaciones que se basan en la búsqueda o revisión a cerca de las aplicaciones biotecnológicas de los microorganismos en diversos ámbitos (LIN, et al., 2017; Barnes, et al., 2018).

\section{Potencial Biotecnológico ambiental}

La realización del presente trabajo, permitió la identificación de microorganismos procedentes de la Reserva biológica El Chcoyero/ElBrujo y del Rio chiquito de León como Pseudomona mendocina, Trichoderma harzianum, los cuales han sido reportados como útiles en la biorremediación en suelos contaminados con furano y pentaclorofenol (Carballo, et al., 2012 ; LIN, et al., 2017; Mendoza, et al.,2010), además se ha reportado microorganismos como Penicillium citrinum y Alcaligenes faecalis subs faecalis (Castro, et al., 2014; Barnes, et al., 2018) como útiles en la degradación de lodos petroquímicos ricos en hidrocarburos aromáticos y saturados. Estos últimos microrganismos, también fueron aislados e identificados en los sitios antes mencionados y se muestran en la Figura 2.

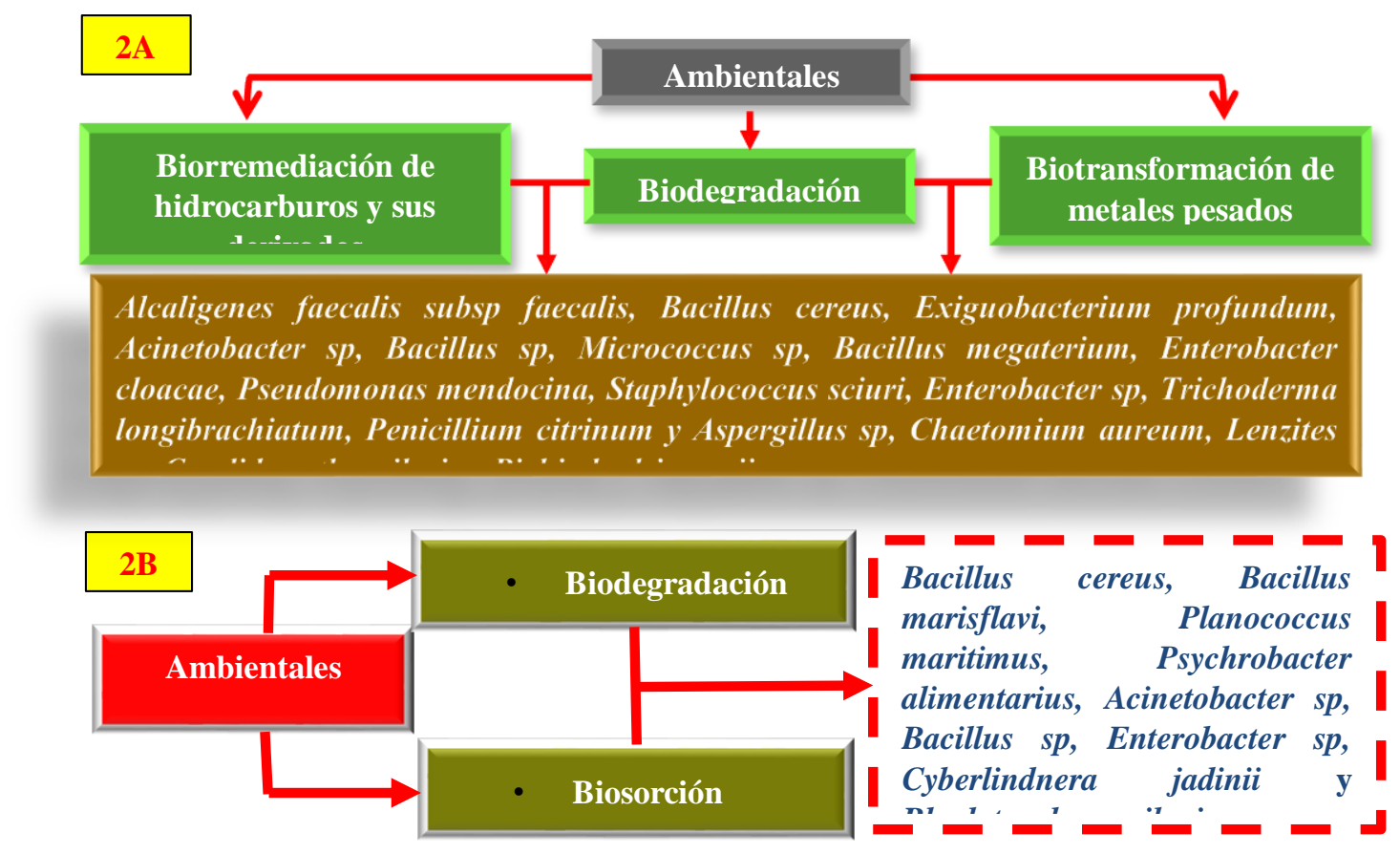

Figura 2. Mediante las figuras 2A y 2B, se puede apreciar una buena cantidad de microorganismos que han sido reportados como útiles para el desarrollo de diversos bioprocesos ambientales. 
En este trabajo se determinó que la bacteria Micrococcus sp y las levaduras Candida orthopsilosis y Pichia kudriavzevii (Figura 3) tienen potencial para degradar colorantes que contaminan el ambiente (Du, et al., 2013). Otros microrganismos como Acinetobacter sp y Enterobacter sp se han reportado como útiles en la biodegradación de ácidos orgánicos como ácido Tánico y fenol (Wang, et al., 2007). El desarrollo de estos trabajos en bioprospección microbiana en la búsqueda de microorganismos útiles para el desarrollo de bioprocesos aplicables al ambiente, aportó una gama de microorganismos plenamente identificados (faltando aún un buen grupo por identificar) y que han sido reportados en la literatura científica como útiles para el desarrollo de diversos bioprocesos como: La remoción de metales pesados como $\mathrm{Cd}, \mathrm{Pb}, \mathrm{Zn}, \mathrm{Cr}, \mathrm{Cu}$, As, y Hg del ambiente a través de biosorción, biorremoción y biorremediación, para lo cual se han identificado por medio de este trabajo microorganismos como Bacillus cereus, Bacillus megaterium, Enterobacter cloacae, Pseudomonas mendocina, Staphylococcus sciuri, Acinetobacter sp, Enterobacter sp, Micrococcus sp, Penicillium citrinum, Chaetomium aureum, Lenzites sp y Pichia kudriavzevii (Rosu, et al., 2019; Ilyas and Rehman, 2018). Para la biorremediación de hidrocarburos y sus derivados se han identificado por este trabajo, microorganismos como Alcaligenes faecalis subsp faecalis, Bacillus cereus, Exiguobacterium profundum, Acinetobacter sp, Bacillus sp, Micrococcus sp, Trichoderma longibrachiatum, Penicillium citrinum y Aspergillus sp (Barnes, et al., 2018; Lustosa, et al., 2018). Para la degradación de colorantes en aguas residuales mediante los mecanismos de biosorción, biodegradación o biorremediación se identificaron microorganismos como Micrococcus sp, Candida orthopsilosis y Pichia kudriavzevii (Muñoz-Silva, et al., 2019; Sinha, et al., 2018). Lo anterior, abre paso a la realización de diversas investigaciones que podrían contribuir a la preservación y cuidado del ambiente por medio de la biotecnología ambiental.

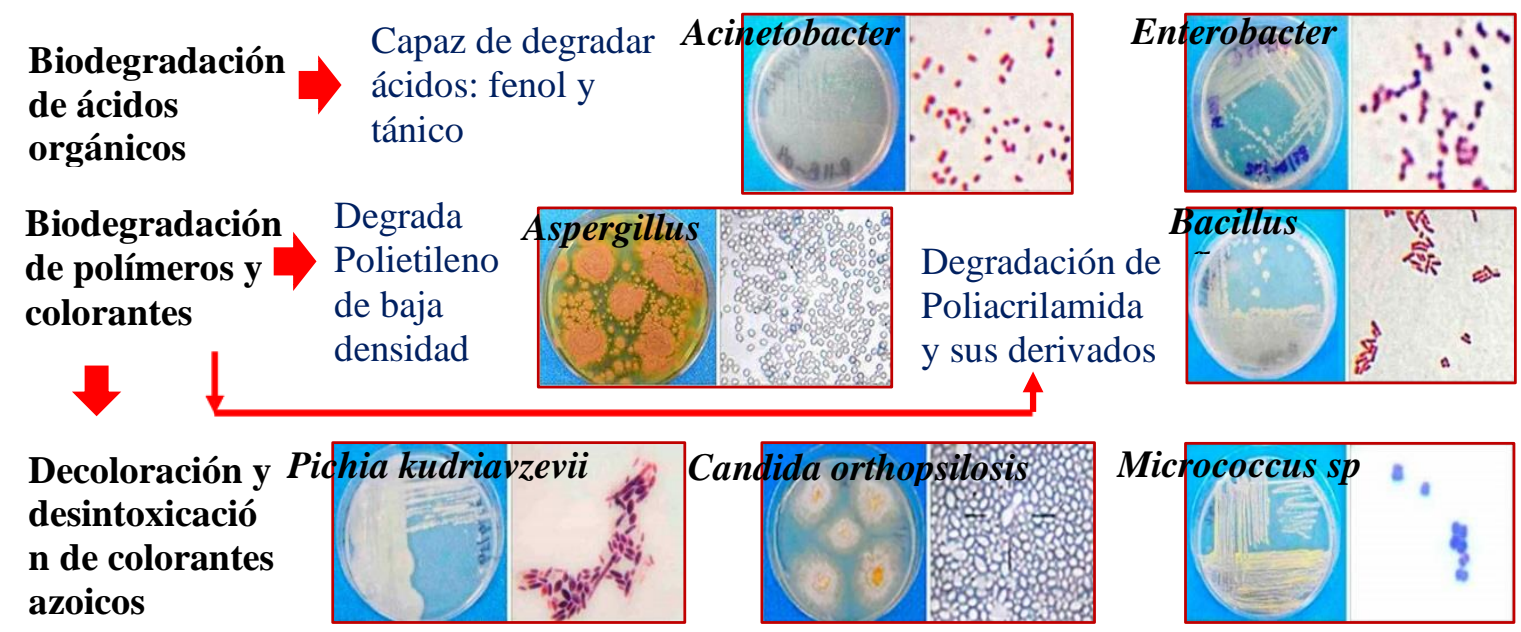

Figura 3. Se muestra una pequeña parte de los aislados de hongos filamentosos y bacterias, obtenidos durante el proceso de aislamiento y purificación del muestreo realizado a la Reserva natural El Chocoyero / El Brujo. Se pueden ver diferentes coloraciones ante PDA (Papa Dextrosa Agar) y ante LB (Luria Bertani) respectivamente, así como diferentes formas de conidias y respuestas ente la tinción Gram.

\section{Potencial Biotecnológico Agrícola}

Algunas de las aplicaciones más comunes entre la gama de cepas identificadas por medio de los trabajos de Bioprospección desarrollados en el PIENSA-UNI, es la promoción del crecimiento vegetal y el control de plagas en diversos cultivos o en sus cosechas. Entre las bacterias identificadas por Delgado-Silva y FonsecaCruz, 2019 (Figura 4A) y que realizan estas funciones, se destacan: Acinetobacter calcoaceticus, Aeromonas hydrofhila, Enterobacter cloacae, Klebsiella pneumoniae, Pseudomonas aeruginosa, Psychrobacter alimentarius, Serratia marcescens y Terribacillus saccharophilus y entre los hogos el Penicillium 
janthinellum y Penicillium simplicissimum, el género Trichoderma sp, y las levaduras Cyberlindenera jadinii y Rhodotorula mucilaginosa (Saravanakumar, et al., 2017; Grunennvaldt, et al. 2018).

Por otra parte, Ríos-Guevara, (2019), identificó microorganismos promotores del crecimiento vegetal como las bacterias Alcaligenes faecalis subsp faecalis, Bacillus megaterium, Enterobacter cloacae, Pantoea dispersa, Staphylococcus sciuri, Enterobacter sp, Bacillus cereus; los hongos Trichoderma harzianum, Trichoderma longibrachiatum, Chaetomium brasiliense, Penicillium citrinum y Chaetomium aureum; y la levadura Candida orthopsilosis (Saravanakumar, et al., 2017; Grunennvaldt, et al. 2018). Tanto DelgadoSilva y Fonseca-Cruz, (2019), como Ríos-Guevara (2019), identificaron a las bacterias Esterobacter cloacae, Erwinia sp y Staphylococcus sciuri (Akram et al., 2016; Muleta, et al., 2013), como útiles para el desarrollo de la capacidad solubilizadora de fosfato en los suelos que dispongan del mismo en forma no asimilable por la planta (Figura 4B).

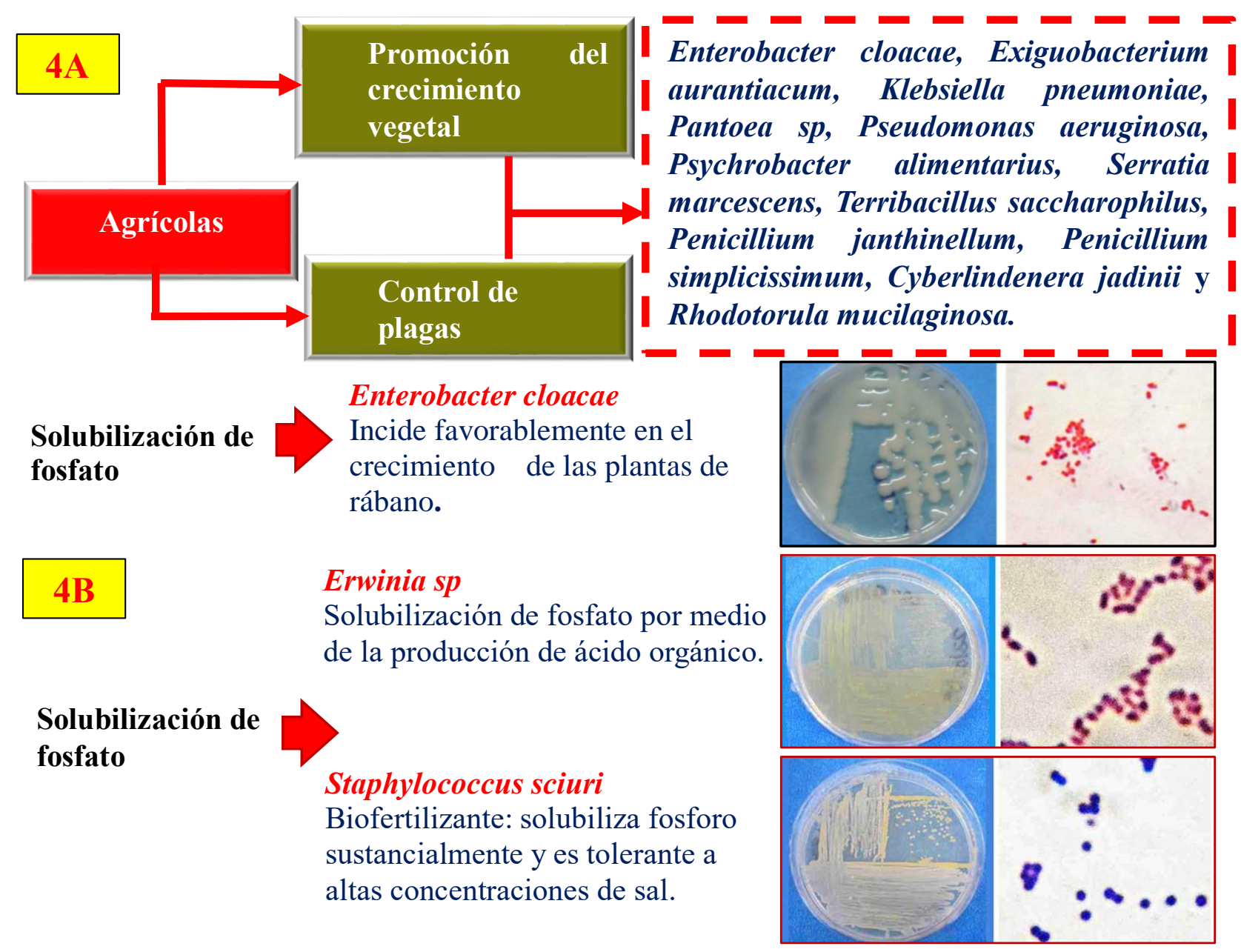

Figura 4. Se resumen los resultados obtenidos por Delgado-Silva y Fonseca-Cruz, 2019, en cuanto a microorganismos de interés en aplicaciones agrícolas, tanto para la promoción del crecimiento vegetal, como para el control de plagas (4A), además de placas Petri conteniendo el cultivo microbiano correspondiente y su respuesta a la tinción Gram, al mismo tiempo se explica la función solubilizadora de fosfato que realizan las mismas.

\section{Potencial Biotecnológico Industrial}

Las aplicaciones industriales más comunes entre la gama de cepas identificadas por medio de los trabajos de Bioprospección desarrollados en el PIENSA-UNI, son la biosíntesis de nanopartículas de oro y plata (Au 
y Ag), por las bacterias: Bacillus marisflavi, Klebsiella pneumoniae y Bacillus sp y los hongos Cyberlindnera jadinii, Trichoderma harzianum Penicillium citrinum, Aspergillus sp y Trichoderma longibrachiatum (Nadaf y Kanase, 2016). También se encontraron algunas especies bacterianas que actúan en la generación de energía como: Aeromonas hydrophila, Bacillus cereus, Enterobacter sp y Klebsiella sp (Du, et al.,2007; Nadaf y Kanase, 2016).

A pesar de la importancia que conlleva la producción de polihidroxialcanoato para la producción de biopolímeros solo se identificaron dos especies que intervienen en el proceso, las bacterias Bacillus cereus y Bacillus megaterium (Delgado-Silva y Fonseca-Cruz, 2019). Por ultimo pero no menos importante, cabe mencionar la gran cantidad de cepas microbianas que tiene capacidad de biosíntesis de enzimas y proteínas (proteasa, celulasa, lipasa, quitinasa, pectinasa, naringinasa, lactasa, amilasa, 26-KDa, ente otras) dentro de las cuales se pueden mencionar las bacterias: Aeromonas hydrophila, Alcaligenes faecalis subsp faecalis, Bacillus sp, Enterobacter sp, Exiguobacterium profundum, Micrococcus sp, Pantoea dispersa, Proteus penneri, Pseudomonas aeruginosa y Serratia marcescens y los hongos: Cyberlindnera jadinii, Trichosporon asahii, Humicola sp, Penicillium citrinum, Penicillium janthinellum, Penicillium simplicissimum, Trichoderma sp, Trichoderma harzianum y Trichoderma longibrachiatum (Sun, et al., 2010; Nguyen, et al., 2014).

El desarrollo del presente trabajo confirmó que algunos de los microorganismos identificados como las bacterias Serratia marcescens y Aeromonas hydrophila y la levadura Trichosporon asahii podrían ser utilizadas en el desarrollo de bioprocesos para la producción de lipasa y por tanto tienen un amplio potencial en la industria orgánica. En este estudio se han encontrado e identificado otros microorganismos que intervienen en la biosíntesis de lipasa como las bacterias Alcaligenes faecalis subsp faecalis, Proteus penneri y Pseudomonas aeruginosa y los hongos Trichosporon asahii y Penicillium simplicissium (Figura $5)$.
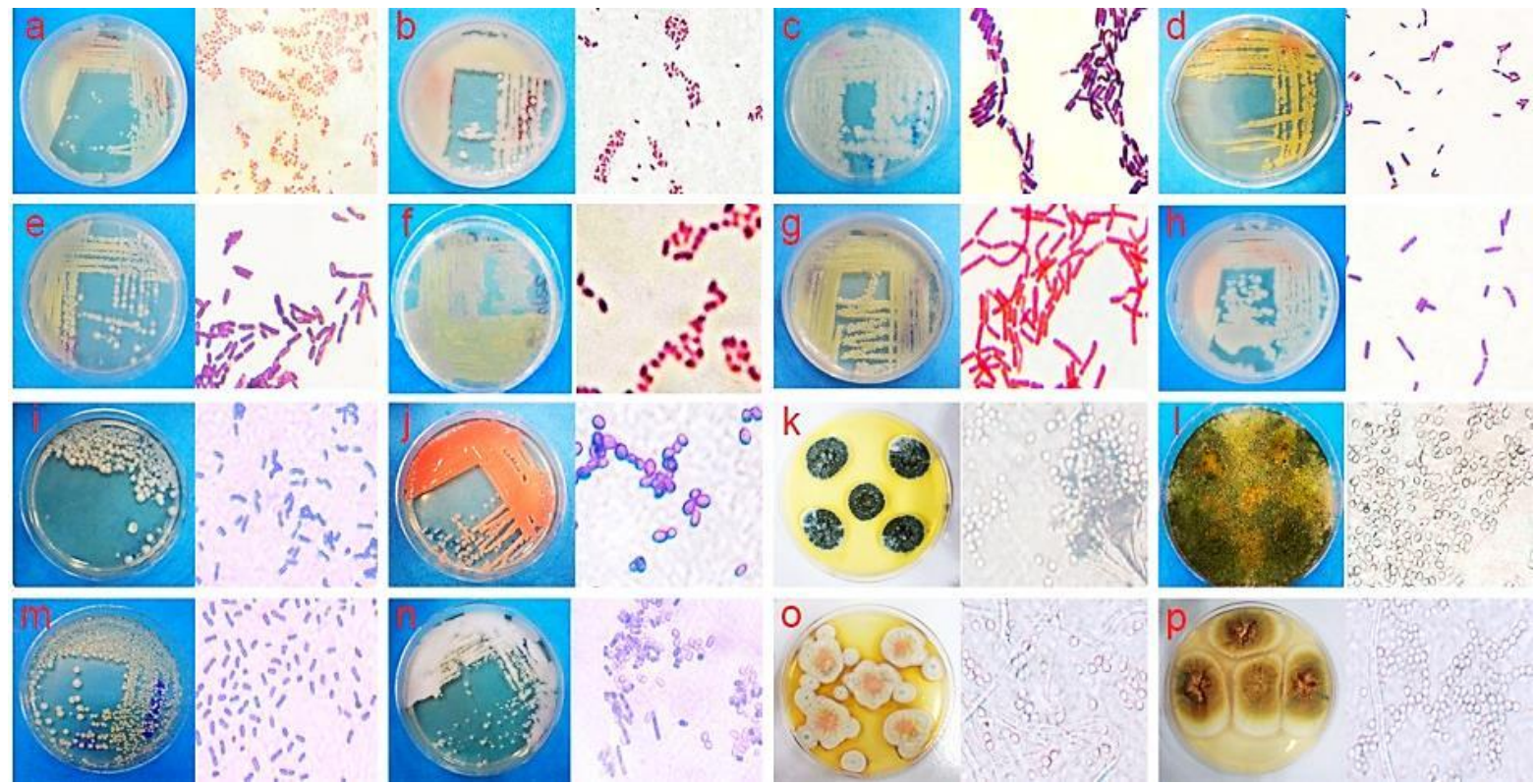

Figura 5. Observación macro y microscópica de algunas de las cepas bacterianas, de hongos levaduriformes y filamentosos, con potencial biotecnológico industrial. (a.) Acinetobacter calcoaceticus. (b) Aeromonas hydrophila. (c) Bacillus cereus. (d). Bacillus marisflavi. (e) Enterobacter sp. (f) Pantoea dispersa. (g) Pseudomonas sp. (h) Bacillus sp. (i) Cybelindnera jadinii. (j) Rhodotorula mucilaginosa. (k). Penicillium citrinum, (l) Trichoderma longibrachiatum. (m) Pichia jadinii. (n) Trichosporon asahii. (o). Penicillium janthinellum. (p) Penicillium simplicissimum. 
Finalmente, los trabajos desarrollados en bioprospección microbiana en búsqueda de microorganismos útiles para el desarrollo de bioprocesos aplicables a la industria, la agricultura y el ambiente, aportan un buen número de microorganismos plenamente identificados y que han sido reportados en la literatura científica como útiles para el desarrollo de diversos bioprocesos. Lo anterior, abre las puertas para el desarrollo de diversas investigaciones que podrían contribuir por medio de la biotecnología al desarrollo de agro y la industria nacional.

\section{CONCLUSIONES}

El desarrollo de estos trabajos en bioprospección microbiana en la búsqueda de microorganismos útiles para el desarrollo de bioprocesos aplicables al ambiente, aportó una gama de microorganismos plenamente identificados (faltando aún un buen grupo por identificar) y que han sido reportados en la literatura científica como útiles para el desarrollo de diversos bioprocesos. Para el desarrollo de sistemas de tratamiento y bioprocesos aplicables a problemas ambientales, se han identificado microorganismos como: Bacillus cereus, Bacillus marisflavi, Planococcus maritimus, Psychrobacter alimentarius, Acinetobacter sp, Bacillus sp, Enterobacter sp, Cyberlindnera jadinii y Rhodotorula mucilaginosa, Bacillus megaterium, Enterobacter cloacae, Pseudomonas mendocina, Staphylococcus sciuri, Enterobacter sp, Micrococcus sp, Penicillium citrinum, Chaetomium aureum, Lenzites sp y Pichia kudriavzevii.

Para la biorremediación de hidrocarburos y sus derivados se han identificado por este medio, microorganismos como: Alcaligenes faecalis subsp faecalis, Bacillus cereus, Exiguobacterium profundum, Acinetobacter sp, Bacillus sp, Micrococcus sp, Trichoderma longibrachiatum, Penicillium citrinum y Aspergillus sp. Para la degradación de colorantes en aguas residuales mediante los mecanismos de biosorción, biodegradación o biorremediación se identificaron microorganismos como: Micrococcus sp, Candida orthopsilosis y Pichia kudriavzevii.

Para la biopromoción del crecimiento vegetal y la bioproteción de los estrés abióticos y bióticos en diversos cultivos o en sus cosechas, se han documentado bioprocesos con bacterias y hongos como: Acinetobacter calcoaceticus, Enterobacter cloacae, Exiguobacterium aurantiacum, Klebsiella pneumoniae, Pantoea sp, Pseudomonas aeruginosa, Psychrobacter alimentarius, Serratia marcescens, Terribacillus saccharophilus, Penicillium janthinellum, Penicillium simplicissimum, Cyberlindenera jadinii, Rhodotorula mucilaginosa, Alcaligenes faecalis subsp faecalis, Bacillus megaterium, Pantoea dispersa, Staphylococcus sciuri, Enterobacter sp, Bacillus cereus, Trichoderma harzianum, Trichoderma longibrachiatum, Chaetomium brasiliense, Penicillium citrinum, Chaetomium aureum y Candida orthopsilosis. Para las industrias de producción de enzimas, pigmentos biodegradables, biolixiviación y producción de aceite se han identificado microorganismos como: Planococcus maritimus, Pseudomonas aeruginosa, Serratia marcescens, Bacillus sp, Penicillium janthinellum, Penicillium simplicissimum, Trichoderma sp, Rhodotorula mucilaginosa y Trichosporon asahii. Aeromonas hydrophila, Alcaligenes faecalis subsp faecalis, Exiguobacterium profundum, Pantoea dispersa, Bacillus sp, Enterobacter sp, Micrococcus sp, Aspergillus sp, Humicola sp, Trichoderma harzianum, Trichoderma longibrachiatum, Penicillium citrinum, Candida orthopsilosis.

\section{AGRADECIMIENTOS}

Los autores desean expresar su gratitud al Programa de Investigación Estudios Nacionales y Servicios Ambientales (PIENSA-UNI) bajo la dirección de las MSc. Larisa Korsak; por haber sido contraparte de este proyecto y en cuyas instalaciones se encuentra el laboratorio de biotecnología, en el cual se desarrolló este trabajo de investigación. Al mismo tiempo desean agradecer a la Universidad Nacional de Ingeniería, quien por medio de su vicerrectoría de Investigación y Desarrollo ha facilitado el aporte financiero para llevar a cabo el proyecto, a todos ellos nuestras mayores muestras de gratitud y compromiso de seguir adelante en 
la búsqueda de resultados que contribuyan a resolver problemas en las áreas agrícolas, ambientales e industriales de Nicaragua, mediante el uso de la biotecnología.

\section{REFERENCIAS}

Aceves, A. y Castañeda, L. (2012). Producción biotecnológica de lipasas microbianas, una alternativa sostenible para la utilización de residuos agroindustriales. Vitae, 19(3), 244-247. ISSN: 0121-4004

Akram, M. S., Shahid, M., Tariq, M., Azeem, M., Javed, M. T., Saleem, S., and Riaz, S. (2016). Deciphering Staphylococcus sciuri SAT-17 Mediated Anti-Oxidative Defense Mechanisms and Growth Modulations in Salt Stressed Maize (Zea mays L.). Frontiers in Microbiology, 7, 1-14. doi:10.3389/fmicb.2016.00867

Blanco, Ch.M.E y Lanza,J.C.A. 2001. Influencia del factor de dispersión en la remoción de coliformes por las lagunas secundarias de estabilización de ARD de la ciudad de Masaya. Tesis para optar al título de maestro en Ingeniería Ambiental y Maestro en Ciencias ambientales respectivamente por cada uno de los autores de este trabajo. UNI-PIDMA.

Barnes, N. M., Khodse, V. B., Lotlikar, N. P., Meena, R. M., and Damare, S. R. (2018). Bioremediation potential of hydrocarbon-utilizing fungi from select marine niches of India. Biotech, 8 (21). ISSN:21905738

Carballo, M. E., Martínez, A., Salgado, I., Maldener, I., Álvarez, M., Boza, A., ...Cruz, M. (2012). Capacidad de captura de cadmio y cinc por bacterias, microalgas y levaduras. Revista Cubana de Ciencias Biológicas, 1(1), 34-43. ISSN: 2307-695X

Castro, R. A., Quecine, M. C., Lacava, P. T., Batista, B. D., Luvizotto, D. M., Marcon, J., Ferreira, A., Melo, I. S., and Azevedo, J. L. (2014). Isolation and enzyme bioprospection of endophytic bacteria associated with plants of Brazilian mangrove ecosystem. SpringerPlus, 3(1), 382. DOI: 10.1186/2193-1801-3-382

Delgado-Silva, H.D y Fonseca Cruz, E.J. (2019). Bioprospección microbiana dirigida a identificar microbiológica y molecularmente microorganismos de utilidad agrícola e industrial en Nicaragua. Trabajo monográfico para optar al título de Ingeniero Químico, presentado a la Facultad de Ingeniería Química de la Universidad Nacional de ingeniería UNI, Julio del 2019.

Du, N. L., Zhao, M., Li, G., Xu, F., Chen, W., and Zhao, Y. (2013). Biodegradation of malachite green by Micrococcus sp strain BD15: Biodegradation pathway and enzyme analysis. International Biodeterioration \& Biodegradation, 78, 108-116. ISSN:0964-8305

Du, L., Jiang, H., Liu, X. and Wang, E. (2007). Biosynthesis of gold nanoparticles assisted by Escherichia coli DH5 $\alpha$ and its application on direct electrochemistry of hemoglobin. Electrochemistry Communications, 9(5), 1165-1170. doi: 10.1016/j.elecom.2007.01.007

Flores-Hernández, M.S y Roque-Castellano, E.V. (2017). Aislamiento y caracterización microbiana (Microbiológica y molecular) en la búsqueda de Bacillus subtilis a partir de bioisnumos comerciales y pruebas de antagonismo frente a hongos Fito patógenos. Trabajo monográfico para optar al título de Ingeniero Agrícola, presentado a la Facultad de Tecnología de la construcción de la Universidad Nacional de ingeniería UNI, marzo del 2017.

González, R. (2011). Biotecnología, Historia y Desarrollo: Situación Actual en Nicaragua. II CONGRESO MULTIDISCIPLINARIO E INTERNACIONAL DE AGROBIOTECNOLOGÍA. Conferencia llevada a cabo en Nicaragua. 
González, T. (2012). Presentación del número extraordinario: Microbiología Industrial y Biotecnología Microbiana.SEM@FORO, 53, 29-60. ISSN: 2254-4399

Guillén-Rodríguez, L.S y Martínez-Laguna, G.I. (2019). Desarrollo del proceso de producción a escala de laboratorio, para el crecimiento de un consorcio microbiano con propiedades biofertilizantes aislados de bioinsumos artesanales. Trabajo monográfico para optar al título de Ingeniero Químico, presentado a la Facultad de Ingeniería Química de la Universidad Nacional de ingeniería UNI, noviembre del 2019.

Grunennvaldt., R. L., Degenhardt-Goldbach, J., De Cássia Tomasi, J., Dos Santos, G. D., Vicente, V. A., and Deschamps, C. (2018). Bacillus megaterium: an endophytic bacteria from callus of Ilex paraguariensis with growth promotion activities. Biotecnología Vegetal, 18(1), 3-13. ISSN 2074-8647

Huete, J. y Orozco, D. (2001). Percepciones sobre biotecnología en Nicaragua. Encuentro, 33(58), 83-90. Doi: https://doi.org/10.5377/encuentro.v0i58.4117

Ilyas, S., and Rehman, A. (2018). Metal resistance and uptake by Trichosporon asahii and Pichia kudriavzevii isolated from industrial effluents. Archives of Environmental Protection, 44(3), 77-84. DOI:10.24425/122291

LIN, J. L., CHEN, S. C., SUNG, W. P., CHEN, T. Y., SURAMPALLI, R. Y., and KAO, C. M. (2017). Bioremediation of OCDF-contaminated soils by novel bacterial strain. Applied Ecology and Environmental Research, 15(3), 713-723. DOI:10.15666/aeer/1503_713723

Loásiga, C. \& Cisne, J. (2001). Recursos genéticos y biotecnología en Nicaragua (1st ed., pp. 18-19). Managua: MARENA.

Lustosa, M. A., López, J. A., Santos, K. C., Padilha, F. F., Hernández-Macedo, M. L., and Cabrera-Padilla, R. Y. (2018). Petroleum hydrocarbon degradation by isolated mangrove bacteria. Revista peruana de biología, 25(4), 453-456. doi:10.15381/rpb.v25i4.15537

Méndez-Úbeda, J.M. (2018). Desarrollo a nivel de laboratorio de un bioplaguicida a base de Bacillus subtilis, para el control de hongos Fito patógenos en cultivos de interés agrícola. Tesis para optar al título de Master en Ciencias de la Biotecnología, presentado a la coordinación de la Maestría Interinstitucional (UNAN-Mga, UNAN-León, UNA y UPOLI) en Biotecnología, marzo del 2018.

Mendoza, J. C., Perea, Y. S., Pretelin, C., Silveti, A., Martínez, M. A., Pérez, G., Arriola, J. (2010). Biosorción de Cromo, Arsénico y Plomo de soluciones acuosas por cultivos bacterianos en suspensión. Revista Latinoamericana el Ambiente y las Ciencias, 1(2), 67-73. ISSN:2007-512X

Muleta, D., Assefa, F., Börjesson, E., and Granhall, U. (2013). Phosphate-solubilising rhizobacteria associated with Coffea arabica L. in natural coffee forests of southwestern Ethiopia. Journal of the Saudi Society of Agricultural Sciences, 12(1), 73-84. ISSN: 1658-077X

Muñoz-Silva, L., Olivera-Gonzales, P., SantillánTorres, M., y Tamariz-Angeles, C. (2019). Microorganismos tolerantes a metales pesados del pasivo minero Santa Rosa, Jangas (Perú). Revista peruana de biología, 26(1), 109-118. doi:10.15381/rpb.v26i1.15914

Nadaf, N. and Kanase, S. (2016). Biosynthesis of gold nanoparticles by Bacillus marisflavi and its potential in catalytic dye degradation. Arabian Journal of Chemistry. doi: 10.1016/j.arabjc.2016.09.020 
Nguyen, A., Huang, C., Liang, T., Nguyen, V., Pan, P. and Wang, S. (2014). Production and purification of a fungal chitosanase and chitooligomers from Penicillium janthinellum D4 and discovery of the enzyme activators. Carbohydrate Polymers, 108(1), 331-337. doi: https://doi.org/10.1016/j.carbpol.2014.02.053.

O’Connor-Mendoza, J.L. (2019). Desarrollo de un consorcio microbiano de aislados autóctonos provenientes de bioinsumos comerciales (Artesanales), con fines de utilización como biofertilizante en cultivos de interés agrícola. Tesis para optar al título de Master en Ciencias de la Biotecnología, presentado a la coordinación de la Maestría Interinstitucional (UNAN-Mga, UNAN-León, UNA y UPOLI) en Biotecnología, noviembre del 2019.

Páramo-Aguilera, L.A., Fonseca-Cruz, E.J., Delgado-Silva, H.D., Cabistán-Calderón, K.E y Rios-Guevara, C.K. 2018. La Bioprospección en Nicaragua: Avances en la búsqueda de aplicaciones agrícolas, industriales y ambientales. Revista científica NEXO, Vol.31, $\mathrm{N}^{\circ} .02$, pp. 89-103. ISSN-E 1995-9516. http//dx.doi.org/10.5377/nexo-v3li2.6833

Pedraza, R., Teixeira, K., Fernández, A., García, I., Baca, B y Azcón, R. (2010). Microorganismos que mejoran el crecimiento de las plantas y la calidad de los suelos. Revisión. Revista Corpoica - Ciencia y Tecnología Agropecuaria, 11(2), 155-164. doi: https://doi.org/10.21930/rcta.vol11 num2 art:206

Piñero, J. (2013). Importancia biotecnológica de la microbiodiversidad. Los nuevos cazadores de microbios. Revista Venezolana de Ciencia y Tecnología de Alimentos, 4(2), 284-317. ISSN: 2218-4384

Ríos-Guevara, C. K. (2019). Bioprospección microbiana para el desarrollo de aplicaciones ambientales. Trabajo monográfico para optar al título de Ingeniero Químico, presentado a la Facultad de Ingeniería Química de la Universidad Nacional de ingeniería UNI, agosto del 2019.

Roșu, C. M., Vochița, G., Mihășan, M., Avădanei, M., Mihai, C. T., and Gherghel, D. (2019). Performances of Pichia kudriavzevii in decolorization, biodegradation, and detoxification of C.I. Basic Blue 41 under optimized cultural conditions. Environmental Science and Pollution Research, 26(1), 431-445. DOI:10.1007/s11356-018-3651-1

Saravanakumar, K., Li, Y., Chuanjin, Y., Wang, Q., Wang, M., Sun, J., ...Chen, J. (2017). Effect of Trichoderma harzianum on maize rhizosphere microbiome and biocontrol of Fusarium Stalk rot. Scientific Reports, 7, 1-13. DOI:10.1038/s41598-017-01680-w

Sinha, A., Lulu, S., V. S., Banerjee, S., Acharjee, S., and Osborne, W. J. (2018). Degradation of reactive green dye and textile effluent by Candida $s p$ VITJASS isolated from wetland paddy rhizosphere soil. Journal of Environmental Chemical Engineering, 6(4), 5150-5159. doi: https://doi.org/10.1016/j.jece.2018.08.004

Sun, H., Ge, X., Hao, Z. and Peng, M. (2010). Cellulase production by Trichoderma sp. on apple pomace under solid state fermentation. African Journal of Biotechnology, 9(2), 163-166. ISSN: 1684-5315

Toruño, V.L y Escobar, V.E. 2002. Influencia del coeficiente de dispersión en la remoción de coliformes fecales en las lagunas de estabilización secundarias de Masaya. Tesis para optar al título Maestro en Ciencias Ambientales. UNI-PIDMA.

Wang, Y., Tian, Y., Han, B., Zhao, H., Bi, J. y Cai, B. (2007). Biodegradation of phenol by free and immobilized Acinetobacter sp. strain PD12. Journal of Environmental Sciences, 19, 222-225. doi: https://doi.org/10.1016/S1001-0742(07)60036-9 


\section{SEMBLANZA DE LOS AUTORES}

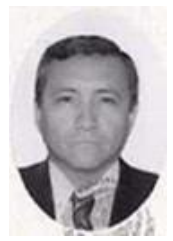

Leandro Alberto Páramo Aguilera, Graduado como Ingeniero Químico con maestría en Ingeniería Química y énfasis en procesos biotecnológicos, en el Instituto Superior Politécnico "José Antonio Echeverría", ISPJAE, de la Ciudad de la Habana, Cuba, en el año 1990. En 1997 se gradúa como Master en Microbiología y énfasis en bacteriología en la Universidad de Costa Rica, UCR. En junio del 2012, se gradúa como Doctor en Ciencias en el área de Biotecnología en el Centro de Biotecnología Genómica (CBG) del Instituto Politécnico Nacional (IPN) de México. Amplia experiencia en el desarrollo de procesos biotecnológicos (biofertilizantes, bebidas alcohólicas, fermentados lácteos, bioprospección, compostaje, etc).

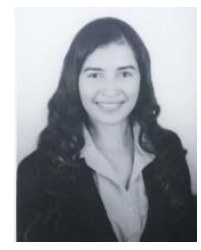

Heysell Dodanig Delgado-Silva: Graduada como Ingeniera Química en la Universidad Nacional de Ingeniería (UNI), donde actualmente es responsable del Laboratorio de Biotecnología del Programa de Investigación Estudios Nacionales y Servicios del Ambiente (PIENSA). Desarrolló proyecto de investigación con interés en el área de biotecnología para el desarrollo de Bioprocesos industriales y agrícolas. Además, es graduada del Instituto Nacional Técnico para la Administración y Economía como Técnico General de Contabilidad, Ingles Básico y Cultura Emprendedora. Ha participado en varios congresos nacionales y latinoamericano en diversas áreas; XXII Congreso Latinoamericano de Estudiantes de Ingeniería y $2^{\mathrm{do}}$ y $3^{\text {er }}$ foro de proyectos de investigación, desarrollo, innovación, posgrado y extensión de la UNI.

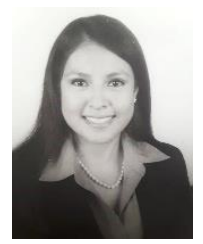

Cesia K. Ríos-Guevara: Graduada como Ingeniería Química de la Universidad Nacional de Ingeniería (UNI). Desarrolló proyecto de investigación de carácter innovador a nivel nacional concernientes a la biotecnología dirigido al desarrollo de procesos medio ambientales, agrícola e industriales del cual ha realizado 3 publicaciones de artículos en diferentes revistas científicas. Ha participado en el $2^{\text {do }}$ y $3^{\text {er }}$ foro de proyectos de investigación, desarrollo, innovación, posgrado y extensión de la UNI. 